\title{
POSSIBLE THERAPEUTIC ROLE OF CHOLINERGIC AGONISTS ON COVID-19 RELATED INFLAMMATORY RESPONSE
}

\author{
Elif Baris ${ }^{1}$, M. Aylin Arici ${ }^{2}$ \\ 1 Dokuz Eylul University Graduate School of Health Sciences; Izmir University of Economics, Faculty of Medicine, \\ Medical Sciences, Department of Pharmacology. \\ 2 Dokuz Eylul University Faculty of Medicine, Department of Medical Pharmacology.
}

\begin{abstract}
Address for Correspondence: Elif Baris E-mail: elif.baris@ieu.edu.tr
Received: 28.01.2021; Accepted: 24.02.2021; Available Online Date: 04.03.2021

(C) Copyright 2021 by Dokuz Eylül University, Institute of Health Sciences - Available online at https://dergipark.org.tr/en/pub/jbachs Cite this article as: Baris E, ARICI M.A Possible Therapeutic Role of Cholinergic Agonists on COVID-19 related inflammatory response. J Basic Clin Health Sci 2021; 1: 102- 108.
\end{abstract}

\begin{abstract}
Severe acute respiratory syndrome-corona virus-2 (SARS-CoV-2) or coronavirus infectious disease (COVID-19) outbreak is continued to spread all over the world recently with the high mortality and morbidity rates. It is also known well COVID-19 is leading causes of acute lung injury and acute respiratory distress syndrome (ARDS), sepsis and multiorgan failure. Current treatment of COVID-19, includes different strategies targeting preventing viral replication or treating secondary infections and decreasing exaggerated immune response. Although antiviral, antimicrobial, immunomodulatory agents including anti-cytokines and glucocorticoids have been currently applied, there is lack of a specific treatment for COVID-19. In this review, possible therapeutic roles of cholinomimetic drugs in the control of COVID-19 related inflammation is discussed.
\end{abstract}

Keywords: Cholinomimetic drugs, COVID-19, SARS-CoV-2, Cytokine Storm

\section{INTRODUCTION}

The corona virus that appeared in Wuhan, China in late 2019, called the SARS-CoV-2 by the World Health Organization. Clinical findings of COVID-19 are variable, coronavirus infectious disease related acute respiratory distress syndrome (ARDS) is the major cause in mortality and morbidity. In the pathogenesis of COVID-19, exaggerated inflammatory response affect the severity of infection because of the host response. Basic mechanism is based on the cytokine storm (1).

Pathogenesis of hyperinflammation in COVID-19 Several pathogens, including bacteria and viruses, might be the cause of systemic inflammation, sepsis and multiple organ failure (2). The pathogenesis of the COVID-19 includes the systemic inflammatory response syndrome and cytokine storm which is the leading cause of sepsis. In patients diagnosed with COVID-19, the presence of viral infection and ARDS indicates the development of sepsis.

Recent studies revealed that the high plasma levels of many cytokine in patients diagnosed with COVID19 such as C-reactive protein (CRP), interleukin (IL)2, IL-6, IL-7, IL-10, tumor necrosis factor alpha (TNF $\alpha)$ interferon gamma (IFN- $\gamma$ ), granulocyte macrophage colony stimulating factor (GM-CSF), monocyte chemoattractant protein 1 (MCP1) and macrophage inflammatory protein 1 alpha (MIP1A) 
(1). It is showed that the highest level of serum IL-10, IL-2, IL-4, TNF $\alpha$ and IFN- $y$ and lowest level of T cell count are detected at 4-6 days of COVID-19. Increased numbers of $T$ cells and decreased inflammatory mediators have been associated with disease resolution (3).

Among these pro-inflammatory cytokines IL- 6 , TNF $\alpha$, IL-1 1 , IL-8 are correlated with the development of acute lung injury and ARDS (4). Cytokine mediated increase in alveolar-capillary permeability develops into ARDS. Studies showed that unrestrained proinflammatory cytokine release in COVID-19 could be one of the major factors that determine the prognosis of the patients (5-7). Recent study showed that patients with COVID-19 admitted in intensive care units (ICU), sepsis is the most frequent complication between the non-survivors (8). These results are confirming that the systemic inflammation and sepsis are contributors of poor prognosis of COVID-19 diagnosed patients $(9,10)$. Besides, preliminary studies revealed that COVID-19 related deaths might be a result of uncontrolled inflammatory response (11).

COVID-19 treatment includes anti-inflammatory and immunomodulatory drugs, in addition to antiviral therapy. Hydroxychloroquine has an antiinflammatory effect by inhibiting IL- 6 and TNF $\alpha$ besides of its antiviral effect. Tocilizumab, another immunomodulatory drug used in the treatment, also has an inhibitory effect on IL-6 which increases in cytokine storm (12). Regarding the hyperinflammatory state, there is lack of information about the effectiveness of well-known antiinflammatory drugs such as glucocorticoids in COVID-19 patients. Although, systemic glucocorticoid use is recommended for severe COVID-19 patients to control cytokine storm up to date $(4,13)$

\section{Cholinergic anti-inflammatory pathway (CAP) and inflammatory response}

Unrestrained release of pro-inflammatory cytokines initiates a systemic inflammatory response which can be regulated by cholinergic system that plays a crucial role in balancing the body's response to inflammation and survival. Our body's controls of inflammation through parasympathetic system is called cholinergic anti-inflammatory pathway (CAP). The CAP is defined as a comprehensive neural mechanism that attenuates pro-inflammatory cytokine release through the vagus nerve and cholinergic receptors. Neuronal part is activation of vagus nerve, whereas non-neuronal part is mainly act on nicotinic receptors expressed on mononuclear phagocytic cells as lymphocytes, macrophages, mast cells, dendritic cells, basophils, microglia (14). Pharmacological activation of cholinergic receptors or electrical stimulation of vagus nerve restraint proinflammatory cytokine release in various in vivo models such as ischemia, pancreatitis, colitis, hemorrhage and sepsis are known (15-17). Most of the studies focused on pharmacological interventions to activate cholinergic receptors, particularly alpha 7 nicotinic acetylcholine receptors ( $\alpha 7 n A C h R)$ expressed on mononuclear phagocytic cells to control the inflammatory status (18). Consequently, $\alpha 7 n A C h R s$ play a major role in cholinergic control of inflammation.

\section{The possible role of CAP in COVID-19}

Inflammatory response activates CAP by two ways. In inflammatory conditions release of cytokines from activated inflammatory cells stimulates vagus nerve or their passage through circumventricular organs to brain activates central autonomic parts (19). Brain activates the hypothalamus-pituitary-adrenal (HPA) axis to generate glucocorticoids, sympathetic nervous system to generate catecholamine and efferent vagus nerve for the release of acetylcholine (ACh). As a part of this pathway, ACh activates $\alpha 7 n A C h R$ receptors expressed on macrophages and anti-inflammatory effect is produced (20).

Angiotensin converting enzyme 2 (ACE2) receptors are main targets of SARS-CoV-2 (21). SARS-CoV-2 attaches to ACE2 receptors which are found on the surface of many human cells such as lungs and it enters to cells. Therefore, reducing the ACE2 expression is a therapeutic strategy to prevent the cellular entry of these viruses. Regarding the interaction between the cholinergic system and ACE2; nicotine shown to have a downregulating effect on ACE2 receptor expression in brain of hypoxic-ischemic brain injured rats and in lungs of patients with pulmonary arterial hypertension suggesting the possible cholinergic control (22). However, this finding has not been investigated in context of viral infections. Besides $\alpha 3, \alpha 4, \alpha 5$, and $\alpha 7$ nicotinic $A C h$ receptors expressed on lung epithelium might be secondary target of SARS-CoV-2 which 
takes part in regulation of perfusion that disrupted in ARDS in COVID-19 patients. Furthermore, macrophage mediated cytokine production in bronchoalveolar fluid is under control of Ach and nicotinic receptors expressed on the cells (23). Recent hypothesis further presumed that nicotine might act against SARS-CoV-2 through its modulatory effect on ACE2 and produce an antiinflammatory effect on hyperinflammatory state in COVID-19 patients than currently employed antiinflammatory agent such as anti-cytokine therapies (24).

Hyperinflammatory state compromises excessive and uncontrolled production of inflammatory cytokines. In vivo studies showed that release of inflammatory mediators can be modulated by exogenous administration of cholinergic agonists. The role of cholinomimetic agents like nicotine, GTS21, Ach esterase inhibitors, choline, phosphatidylcholine, citicoline in various inflammatory diseases including inflammatory bowel diseases, pancreatitis, sepsis, arthritis have been investigated with animal models and clinical research (25-30).

Studies have been supported that GTS-21, a selective $\alpha 7 \mathrm{nAChR}$ agonist, and choline, precursor of $\mathrm{ACh}$ and selective $\alpha 7 \mathrm{nAChR}$ agonist, improved survival rate and organ damage while reducing tissue damage and decreasing cytokine levels in animals with sepsis (31-33). Regarding the cardiovascular changes in case of hyperinflammatory state like sepsis, choline donor citicoline decreased microvascular permeability and inflammatory parameters while improving the hypotension and disseminated intravascular coagulation in septic shock $(34,35)$. Choline reduces elevated serum proteins, lipids and inflammatory mediators via the vagal anti-inflammatory pathway activation and endotoxin-induced mononuclear cell activation in case of sepsis (36). The failure of cholinomimetic therapies in $\alpha 7 n A C h R$ knockout mice confirms that $\alpha 7 n A C h R s$ expressed on mononuclear inflammatory cells are key components that modulate inflammatory cytokine levels (31).

Nicotinic hypotheses suggest that nicotinic receptors might involve the entrance of the virus into body through nicotinic receptors of olfactory neurons and airway epithelium. Additionally, hypothesis proposed that SARS-CoV-2 virus might directly interact with nicotinic receptors and antagonize the immunomodulatory effect of nicotine (38). Structural analyses found that nicotine might inhibit SARS-CoV2 protein and ACE2 receptor interaction at lower concentrations (39). Accordingly docking experiments revealed that SARS-CoV-2 Spike Glycoproteins might interact with toxin-binding site and act as an inhibitor of $\alpha 7 n A C h R s$ which supports the role of dysfunction of nicotinic system in pathogenesis of COVID-19 $(40,41)$. Today, nicotinic hypothesis is not supported by clinical data. Studies based on clinical observations revealed that number of active smokers among the hospitalized COVID-19 patients is much lower than expected. Lower rate of active smokers introduces some questions about possible role of nicotinic system in COVID-19 infection. (37).

\section{Clinical evidence of beneficial effects of cholinergic agonists on inflammation}

Several clinical studies also provide supporting data that cholinergic system activator drugs diminish inflammatory response and scoring of organ dysfunction (SOFA score) in patients with sepsis (29, $30,42,43)$.

Among the cholinergic agonists, nicotine has been shown to improve cognitive functions in several conditions including Alzheimer and Parkinson diseases however its therapeutic use is limited due to its unwanted effects mediated by variety of nicotinic receptors such as $\alpha 5, \alpha 3, \alpha 4$, or $\beta 2$ subtype containing nicotinic receptors. Since $\alpha 7 n A C h R s$ mediates the anti-inflammatory effects of cholinergic agonists; selective receptor modulators with lesser autonomic side effects have been developed. Similar with nicotine, $\alpha 7 n A C h R$ agonist GTS-21 has been shown to improve reduce inflammation in patients with osteoarthritis and ulcerative colitis (44). CNI1493 is a compound that acts on intracellular inflammatory pathways via MAP kinase and studies revealed that its activity depends on vagal activation. CNI-1493 attenuates inflammatory response in patients with inflammatory bowel diseases by decreasing TNF a level (45).

Regarding the effect of cholinomimetic agents and hyperinflammatory states like sepsis, transdermal nicotine administration significantly improved endotoxin induced febrile response, change in blood 
pressure and heart rate. Nicotine administration increased plasma cortisol levels along with decreasing both TNF $\alpha$ and IL- 6 levels and increasing anti-inflammatory IL-10 levels (43). Pretreatment with orally administered GTS-21 did not diminish TNF $\alpha$, IL-6, and IL-1 receptor antagonist levels significantly in double-blind placebo-controlled study. However, it produced dose dependent anti-inflammatory effect between treatment groups. Major limitation of this study was the number of volunteers that antiinflammatory effect might be different in larger group of subjects (30).

Clinical studies revealed that acetylcholinesterase inhibitor physostigmine improved SOFA score and mortality rate in patients with perioperative sepsis and septic shock $(17,29,42)$. Rapid recovery in heart rate changes and low dose norepinephrine dose need in patients with perioperative sepsis and septic shock treated with physostigmine have been indicated improved hemodynamic stability. Besides, procalcitonin, C-reactive protein (CRP) and IL-6 levels diminished with physostigmine treatment (17). Recent study revealed that GTS-21 improved lung function along with the reduction in plasma IL-6 level in patients with chronic obstructive pulmonary disease (46). Additionally, $\alpha 7 n A C h R$ positive allosteric modulator and well-known antiparasitic drug ivermectin has been shown to reduce viral replication effectively in-vitro. Further studies might be conducted to reveal its anti-viral activity along with interaction with nicotinic anti-inflammatory pathway $(49,50)$. In a systematic analysis revealed that prevalence of smoking is lower in severe COVID-19 patients hospitalized in China and the US. Novel cross-sectional study in France has been revealed that having severe SARS-CoV-2 infection is less likely in smokers compared to non-smokers which might be due to the anti-inflammatory effect of nicotine (47). Activation of CAP or modulation of angiotensin system with nicotine might be the reason of this situation and pharmaceutical formulations of nicotine in SARS-CoV-2 infection needs to further clinical investigations (48). Besides, by activation of nicotinic anti-inflammatory pathway, decrease of wide range of inflammatory cytokines might be better option to treat COVID-19 related cytokine storm compared to single cytokine inhibitors. All this information suggests that nicotinic agonists acting through the cholinergic anti-inflammatory pathway may be useful in the treatment of COVID-19.
Immunosuppression with corticosteroids has been shown to increase replication of respiratory viruses and promote secondary bacterial infection. Besides glucocorticoids have serious adverse effects even in short term use affecting multiple systems together with immunosuppression, exacerbation of active infections. Immunosuppressive agent hydroxychloroquine has been failed to show therapeutic effect as well as with serious cardiotoxic effects in COVID-19 patients (4).

\section{Conclusion}

Collecting evidence pointed out that hyperinflammation characterized by unrestrained release of inflammatory cytokines leads multiple organ failure and sepsis triggered by viral infections. However, effective treatment for COVID-19 patients with cytokine storm is still an issue. Accumulating evidence support the possible effect of cholinomimetic agents in treatment of COVID-19 patients. Cholinergic agonists might have supportive adjuvant value in treating COVID-19 induced hyperinflammatory state. Although their therapeutic effect is not clear in COVID-19 patients; earlier studies point out that; besides their anti-inflammatory effect nicotinic agonist might interrupt SARS-CoV-2 protein interaction with ACE2 and nAChR that contributes pathophysiology of COVID-19. Agents act through $\alpha 7 n A C h R s$ might be investigated with further randomized controlled studies in order to reveal the possible therapeutic or prophylactic effect along with their safety in patients with novel coronavirus disease.

Financial Disclosure: Grant has not been taken for the preparation of review article.

Peer-review: Externally peer-reviewed.

\section{REFERENCES}

1. Tay MZ, Poh CM, Rénia L, MacAry PA, Ng LFP. The trinity of COVID-19: immunity, inflammation and intervention. Nat Rev Immunol. Published online 2020:1-12.

2. Chaudhry $\mathrm{H}$, Zhou J, Zhong $\mathrm{Y}$, et al. Role of cytokines as a double-edged sword in sepsis. In Vivo. 2013;27(6):669-684.

3. Liu J, Li S, Liu J, et al. Longitudinal characteristics of lymphocyte responses and cytokine profiles in the peripheral blood of SARS-CoV-2 infected patients. EBioMedicine. Published online April 18, 2020:102763. 
4. Zhang W, Zhao Y, Zhang F, et al. The use of antiinflammatory drugs in the treatment of people with severe coronavirus disease 2019 (COVID19): The experience of clinical immunologists from China. Clin Immunol. 2020;214(March):108393.

5. Mehta $\mathrm{P}$, Mcauley DF, Brown $\mathrm{M}$, et al. Correspondence COVID-19: consider cytokine storm syndromes and immunosuppression. Lancet. 2020;6736(20):19-20.

6. Huang $\mathrm{C}$, Wang $\mathrm{Y}$, Li X, et al. Clinical features of patients infected with 2019 novel coronavirus in Wuhan, China. Lancet. 2020;395(10223):497506.

7. Conti $\mathrm{P}$, Ronconi $\mathrm{G}$, Caraffa $\mathrm{A}$, et al. Induction of pro-inflammatory cytokines (IL-1 and IL-6) and lung inflammation by COVID-19: antiinflammatory strategies. J Biol Regul Homeost Agents. 2020;34.

8. Zhou F, Yu T, Du R, et al. Clinical course and risk factors for mortality of adult inpatients with COVID-19 in Wuhan, China: a retrospective cohort study. Lancet. 2020;395(10229):10541062.

9. Zhou $Y, F u$ B, Zheng $X$, et al. Pathogenic T cells and inflammatory monocytes incite inflammatory storm in severe COVID-19 patients. Natl Sci Rev. Published online 2020.

10. Gruner L. Covid-19 Illnes in Native and immunosuppressed states. Lung. 2020;21(1):2225.

11. Ruan Q, Yang K, Wang W, Jiang L, Song J. Clinical predictors of mortality due to COVID-19 based on an analysis of data of 150 patients from Wuhan, China. Intensive Care Med. 2020;46(5):846-848.

12. Geng Y-J, Wei Z-Y, Qian H-Y, Huang J, Lodato R, Castriotta RJ. Pathophysiological Characteristics and Therapeutic Approaches for Pulmonary Injury and Cardiovascular Complications of Coronavirus Disease 2019. Cardiovasc Pathol. 2020;47:107228.

13. Russell $B$, Moss $C$, Rigg A, Hemelrijck M Van. COVID-19 and treatment with NSAIDs and corticosteroids : should we be limiting their use in the clinical setting ? ecancer. 2020;14(1023):1-3.

14. Fujii T, Mashimo M, Moriwaki $Y$, et al. Expression and Function of the Cholinergic System in Immune Cells. Front Immunol. 2017;8:1085.

15. van Westerloo DJ, Giebelen IA, Florquin S, et al. The Vagus Nerve and Nicotinic Receptors
Modulate Experimental Pancreatitis Severity in Mice. Gastroenterology. 2006;130(6):1822-1830.

16. McGrath J, McDonald JWD, MacDonald JK. Transdermal nicotine for induction of remission in ulcerative colitis. Cochrane Libr. 2004;18(4):CD004722.

17. Pinder N, Bruckner T, Lehmann M, et al. Effect of physostigmine on recovery from septic shock following intra-abdominal infection - Results from a randomized, double-blind, placebo-controlled, monocentric pilot trial (Anticholium $®$ per Se). J Crit Care. 2019;52:126-135.

18. Ulloa L. The anti-inflammatory potential of selective cholinergic agonists. Shock. 2011;36(1):97-98.

19. Buller K. Role of circumventricular organs in proinflammatory cytokine-induced activation of the hypothalamic-pituitary-adrenal axis. Clin Exp Pharmacol Physiol. 2001;28:581-589.

20. Kanashiro A, Sônego F, Ferreira $R$, et al. Therapeutic Potential and Limitations of Cholinergic Anti-Inflammatory Pathway in Sepsis. Pharmacol Res. 2017;117:1-8.

21. Lan J, Ge J, Yu J, et al. Structure of the SARSCoV-2 spike receptor-binding domain bound to the ACE2 receptor. Nature. Published online 2020.

22. Oakes JM, Fuchs RM, Gardner JD, Lazartigues $E$, Yue $X$. Nicotine and the renin-angiotensin system. Am J Physiol Regul Integr Comp Physiol. 2018;315(5):R895-R906.

23. Pavlov VA, Tracey KJ. The vagus nerve and the inflammatory reflex--linking immunity and metabolism. Nat Rev Endocrinol. 2012;8(12):743-754.

24. Changeux J, Amoura Z, Rey F, Miyara M. A nicotinic hypothesis for Covid-19 with preventive and therapeutic implications. Qeios. 2020;2:1-11.

25. Aldhous M, Prescott R, Roberts S, Samuel K, Waterfall M, Satsangi J. Does Nicotine Influence Cytokine Profile and Subsequent Cell Cycling/Apoptotic Responses in Inflammatory Bowel Disease? Inflamm Bowel Dis. 2008;14:1469-1482.

26. Gorelick F, Lerch M. Do Animal Models of Acute Pancreatitis Reproduce Human Disease? Cell Mol Gastroenterol Hepatol. 2017;4(2):251-262.

27. Treede I, Braun A, Sparla R, et al. Antiinflammatory effects of phosphatidylcholine. J Biol Chem. 2007;282(37):27155-27164. 
28. Hartmann P, Szabó A, Eros G, et al. Antiinflammatory effects of phosphatidylcholine in neutrophil leukocyte-dependent acute arthritis in rats. Eur J Pharmacol. 2009;622:58-64.

29. Zimmermann JB, Pinder N, Bruckner $\mathrm{T}$, et al. Adjunctive use of physostigmine salicylate (Anticholium $®$ ) in perioperative sepsis and septic shock: Study protocol for a randomized, doubleblind, placebo-controlled, monocentric trial (Anticholium ${ }^{\circledR}$ per Se). Trials. 2017;18(1):1-10.

30. Kox M, Pompe JC, Gordinou De Gouberville MC, Van Der Hoeven JG, Hoedemaekers CW, Pickkers P. Effects of the $\alpha 7$ nicotinic acetylcholine receptor agonist gts-21 on the innate immune response in humans. Shock. 2011;36(1):5-11.

31. Parrish WR, Rosas-Ballina M, GallowitschPuerta M, et al. Modulation of TNF release by choline requires alpha7 subunit nicotinic acetylcholine receptor-mediated signaling. Mol Med. 2008;14(9-10):567-574.

32. Ilcol Y, Yilmaz Z, Cansev M, H. Ulus I. Choline or CDP-choline alters serum lipid responses to endotoxin in dogs and rats: Involvement of the peripheral nicotinic acetylcholine receptors. Shock. 2009;32.

33. Pavlov VA, Ochani M, Yang LH, et al. Selective a7-nicotinic acetylcholine receptor agonist GTS21 improves survival in murine endotoxemia and severe sepsis. Crit Care Med. 2007;35(4):11391144.

34. Schmidt K, Frederick Hernekamp J, Doerr M, et al. Cytidine-5-diphosphocholine reduces microvascular permeability during experimental endotoxemia. BMC Anesthesiol. 2015;15:114122.

35. Yilmaz Z, Ilcol $\mathrm{Y}$, Cansev $\mathrm{M}$, Eralp Inan $\mathrm{O}$, Kocatürk M, H Ulus I. Choline or CDP-choline attenuates coagulation abnormalities and prevents the development of acute disseminated intravascular coagulation in dogs during endotoxemia. Blood Coagul Fibrinolysis. 2010;21(4):339-348.

36. Ilcol YO, Yilmaz Z, Ulus IH. Endotoxin alters serum-free choline and phospholipid-bound choline concentrations, and choline administration attenuates endotoxin-induced organ injury in dogs. Shock. 2005;24(3):288-293.

37. Gonzalez-Rubio J, Navarro-Lopez C, LopezNajera E, et al. Cytokine Release Syndrome (CRS) and Nicotine in COVID-19 Patients: Trying to Calm the Storm. Front Immunol. 2020;11(June):4-7.

38. Changeux J-P. A nicotinic hypothesis for Covid19 with preventive and therapeutic implications. Comptes Rendus Biol. 2020;343:1-7.

39. C SK, Kumar SA, Wei H. A computational insight of the improved nicotine binding with ACE2SARS-CoV-2 complex with its clinical impact. Published online 2020:1-11.

40. Lagoumintzis G, Chasapis C, Alexandris N, et al. COVID-19 and Cholinergic Anti-inflammatory Pathway: In silico Identification of an Interaction between $\alpha 7$ Nicotinic Acetylcholine Receptor and the Cryptic Epitopes of SARS-CoV and SARSCoV-2 Spike Glycoproteins. Published online August 22, 2020.

41. Farsalinos K, Eliopoulos E, Leonidas DD, Papadopoulos GE, Tzartos S, Poulas K. Nicotinic cholinergic system and covid-19: In silico identification of an interaction between sars-cov2 and nicotinic receptors with potential therapeutic targeting implications. Int $\mathrm{J}$ Mol Sci. 2020;21(16):1-15.

42. Pinder N, Zimmermann JB, Gastine S, et al. Continuous infusion of physostigmine in patients with perioperative septic shock: A pharmacokinetic/pharmacodynamic study with population pharmacokinetic modeling. Biomed Pharmacother. 2019;118(June):109318.

43. Wittebole X, Hahm S, Coyle SM, Kumar A, Calvano SE, Lowry SF. Nicotine exposure alters in vivo human responses to endotoxin. Clin Exp Immunol. 2007;147(1):28-34.

44. Pullan RD, Rhodes J, Ganesh S, et al. Transdermal Nicotine for Active Ulcerative Colitis. N Engl J Med. 1994;330(12):811-815.

45. Kosek E, Altawil R, Kadetoff D, et al. Evidence of different mediators of central inflammation in dysfunctional and inflammatory pain--interleukin8 in fibromyalgia and interleukin-1 $\beta$ in rheumatoid arthritis. J Neuroimmunol. 2015;280:49-55.

46. Douaoui S, Djidjik R, Boubakeur M, et al. GTS21 , an $7 \mathrm{nAChR}$ agonist, suppressed the production of key inflammatory mediators by PBMCs that are elevated in COPD patients and associated with impaired lung function. Immunobiology. 2020;(April):151950.

47. Miyara M, Tubach F, POURCHER V, et al. Low incidence of daily active tobacco smoking in 
patients with symptomatic COVID-19. Qeios.

Published online 2020:1-13.

48. Farsalinos K, Barbouni A, Niaura R. Systematic review of the prevalence of current smoking among hospitalized COVID-19 patients in China: could nicotine be a therapeutic option? Intern Emerg Med. 2020;(0123456789).

49. Caly L, Druce JD, Catton MG, Jans DA, Wagstaff KM. The FDA-approved drug ivermectin inhibits the replication of SARS-CoV-2 in vitro. Antiviral Res. 2020;178:104787.

50. Krause RM, Buisson B, Bertrand $S$, et al. Ivermectin: A positive allosteric effector of the $\alpha 7$ neuronal nicotinic acetylcholine receptor. Mol Pharmacol. 1998;53:283-294. 